\title{
Response of a conical, laminar premixed flame to low amplitude acoustic forcing-A comparison between experiment and kinematic theories
}

\author{
Nader Karimi ${ }^{1 *}$ \\ ${ }^{1}$ School of Engineering, University of Glasgow, Glasgow, United Kingdom
}

\begin{abstract}
This paper presents an experimental study on the dynamics of a ducted, conical, laminar premixed flame subjected to low amplitude acoustic excitation from upstream. The heat release response of the flame to velocity disturbances is investigated through measurement of the so called 'flame transfer function' for a wide range of forcing frequencies. The results are compared with those predicted by the existing linear kinematic theories. It is observed that these theories are in general agreement with the experiment, although there exist some disparities. A detailed comparison of the experimental data with the kinematic theories shows that the phase speed of flame disturbances has an essential influence upon the level of agreement between the theory and experiment. The data set presented in this work complements that reported in an earlier study. In keeping with others, visualisation of the excited flames clearly shows that the flame response includes waves on the flame front which are formed at the base and then convect along the flame.
\end{abstract}

Keywords: Laminar premixed flames, Thermoacoustics, Flame transfer function.

\section{Introduction}

Combustion is expected to dominate power generation and propulsion for the foreseeable future. Intensive research activities are, therefore, being carried out across the world to mitigate the environmental issues associated with the combustion of various fuels [1]. In particular, significant attention has been paid to the reduction of combustion generated air pollutants [2]. To comply with the strict air pollution standards, gas turbines must have minimal NOx emission [2,3]. As a result, the gas turbine industry is moving towards utilisation of lean premixed flames to reduce NOx formation [4]. Lean premixed combustion, although significantly improves NOx emission, is prone to a number of combustor instabilities which include thermoacoustic instabilities [5]. In general, occurrence of these instabilities has hindered the development of lean premixed and pre-vaporised gas turbine combustors [4]. Understanding and suppression of thermoacoustic instabilities are, therefore, an ongoing concern for industry and academia [6,7].

Thermoacoustic instabilities are, usually, the result of a complex coupling between the combustion chamber acoustics and unsteady flame heat release. This leads to the generation of strong pressure waves [7] which, in turn, can result in significant hardware damage, flame blow-out or flashback and loss of control of the system

\footnotetext{
*Corresponding author, email address:Nader.Karimi@glasgow.ac.uk
} 
[4]. Importantly, thermoacoustic instabilities may happen in a wide variety of continuous combustion systems including gas turbines, aero-engines, boilers and industrial burners [3]. Consequently, these instabilities are regarded as a major barrier against the wide utilisation of lean premixed, gas turbine combustion [5]. For more than half a century, a substantial amount of effort has been put on understanding, prediction and suppression of thermoacoustic instabilities and the research in this area is ongoing [3]. Yet, there is currently no comprehensive predictive design tool available to identify and eliminate thermoacoustic instabilities at the design stage of combustion systems [4]. As a result, the design of stable combustors relies chiefly on the manufacturer's knowledge and trial and error, which sometimes involves full scale tests [4].

It has been shown by many authors that thermoacoustic instabilities are heavily dependent upon the configuration of the combustion system and, the flame and flow characteristics [5]. In premixed combustors, the most challenging and unknown part of the problem is the response of the premixed flames to acoustic waves [6]. The difficulty, in part, stems from the current lack of a comprehensive understanding on the dynamics of premixed flames [8]. Further, the essential physics of thermoacoustic instabilities are not entirely understood $[6,7]$. This is mostly due to the complexities involved in the interactions between premixed flames and sound waves $[9,10]$ and, to some extent, the effects of system boundaries [11,12].

In practice, it is essential to predict the onset of instability under varying operating conditions. To achieve this, the classical linear stability analysis is, conventionally, conducted through combining the linear flame dynamics with a model of chamber acoustics [6]. This is often regarded as low order modelling and leads to the determination of thermoacoustic stability margins of the systems. In general, low order models are expected to represent the most essential dynamical characteristics of the system, while being simple and easy to use [4]. Detailed understanding of linear flame dynamics is therefore crucial in devising low order flame models and prediction of combustor stability [6]. In recent years, however, it has been demonstrated that thermoacoustic systems can feature strong nonlinear dynamical behaviours [13,14]. These nonlinearities may introduce new stability limits and complicate the stability analysis $[15,16]$.

Laminar flames are often investigated experimentally [17], theoretically [18] and numerically [19,20] to obtain fundamental understanding on the essential physics of flame and sound wave interactions. The resultant physical insight is mostly transferable to more complex cases which include turbulent flames [21]. Experimentally, these analyses are usually performed through exciting a premixed laminar flame by acoustic waves generated by a loud speaker, e.g. [17, 22, 23]. Response of the flame is characterised by the velocity modulations just upstream of the flame and the perturbations in the instantaneous flame heat release [17]. In the linear limit, a transfer function is then defined as the ratio of the disturbances in heat release and upstream mixture velocity, respectively normalised by the mean heat release and mixture velocity [5]. This transfer function serves as the dynamic model of the flame in combustor models [6]. It has been also shown that the interaction of acoustic waves with laminar flames can result in further generation of sound [24]. This secondary generation of acoustic waves may, itself, contribute with the thermoacoustic instability of the combustor.

Theoretical investigations of the dynamics of laminar premixed flames usually make use of the 'G equation' [5]. In this approach, the kinematic evolution of the flame front is related to fluctuations of the flame surface 
area and flame heat release. Fleifil et al. [25] solved the linearised G equation for a laminar flame stabilised in a simple duct flow and developed a theoretical flame transfer function. Later, Ducruix et al. [22] extended this solution to open conical flames. The amplitude of their predicted flame transfer function was in a reasonable agreement with experiment, but the agreement in phase was not as good. This theory was improved by Schuller et al. [23], who assumed convection of flame disturbances along the flame, and obtained better phase agreements with the experiment. The transfer function of Schuller et al. [23] is expressed as

$$
\begin{aligned}
\frac{q^{\prime} /|q|}{u^{\prime} /|\bar{u}|}= & \frac{2}{S t^{2}} \frac{1}{\left(1-\cos ^{2} \alpha\right)} \times \\
& {\left[1-\exp (j S t)+\frac{\exp \left(j S t \cos ^{2} \alpha\right)-1}{\cos ^{2} \alpha}\right] }
\end{aligned}
$$

where $S t=(\omega R) /\left(S_{L} \cos \alpha\right)$. Here, the terms $q$ and $\bar{q}$ are respectively the fluctuating and mean flame heat release (W) and, $u$ and $\bar{u}$ are the fluctuating and mean velocity immediately upstream of the flame (m/s). Further, $S t$ is a Strouhal number and $\omega, R, S_{L}, \alpha$ and $j$ are respectively the forcing frequency $(\mathrm{rad} / \mathrm{s})$, flame holder radius $(\mathrm{m})$, the laminar flame speed $(\mathrm{m} / \mathrm{s})$, the flame half apex angle and the unit imaginary number $(\sqrt[2]{-1})$. Flame disturbances are the surface waves generated at the flame base which then convect downstream towards the flame tip. Phase speed of the flame disturbance is further defined as the convective velocity of the surface wave. This theory assumes that flame disturbances are convected downstream by the mean, cold flow velocity. Schuller et al. [23] observed some disparities between the theoretical phase response in Eq. (1) and experiment at higher frequencies. This model was later verified experimentally by Karimi et al. [17]. In their experiment a ducted flame was subjected to harmonic downstream travelling acoustic disturbances with varying amplitude and frequency [17]. Karimi et al. found that Schuller's linear theory matched their experimental results generally well [17]. This agreement, however, was limited to low amplitude forcing. At higher forcing amplitudes, the flame response was significantly different to that predicted by Eq. (1) [17]. Karimi et al., further, measured the phase speed of flame disturbances and showed that this quantity varies along the flame and depends upon the forcing frequency [17]. A similar conclusion was made by Birbaud et al. [26, 27] in their investigation of free jets and conical flames. Formation of travelling waves on the flames by acoustic forcing has been further confirmed by Kornilov et al. [28, 29]. Furthermore, it has been experimentally shown that the presence of an enclosure [30], and also flame anchoring dynamics [31] may affect the flame dynamics.

More recently, the kinematic model of Schuller et al. [23] was extended to non-uniform flow disturbances by Preetham et al. [32]. In this theory the phase speed of the flame disturbances is not necessarily the same as the mean flow speed and can vary freely [32]. Yet, in the theory of Preetham et al. [32] the value of phase speed remains frequency independent and constant along the flame. The resultant flame transfer function is expressed as [32]

$$
\frac{\dot{q} /|\bar{q}|}{\dot{u} /|\bar{u}|}=2\left(\frac{\eta\left(1-e^{i S t_{2}}\right)+e^{i \eta S t_{2}}-1}{\eta(1-\eta) S t_{2}{ }^{2}}\right) \text {, }
$$


where $S t_{2}=\frac{S t}{\alpha}=\frac{S t\left(1+\beta^{2}\right)}{\beta^{2}}, \beta=\frac{L_{f}}{R}, \alpha=\frac{\beta^{2}}{\beta^{2}+1}, \eta=K \alpha, K=\frac{u_{0}}{u_{c}}$. Further, $u_{0}, u_{c}, L_{f}$ and $R$ are respectively the flow bulk velocity at the flame base $(\mathrm{m} / \mathrm{s})$, flame disturbance phase speed $(\mathrm{m} / \mathrm{s})$, steady flame length $(\mathrm{m})$ and flame base diameter $(\mathrm{m})$.

The analytical analysis of flame dynamics has been, recently, extended to the time domain by Blumenthal et al. [33]. These authors derived expressions for the temporal response of two different flame configurations subjected to an impulse [33]. Through spectral analysis of their temporal impulse response, Blumenthal et al. [33] restored the previously derived flame transfer functions [23]. In another recent work, Kashinath et al. [34] computed the flame phase speed through direct numerical simulation of an acoustically forced, conical laminar flame. Their results clearly show that the phase speed of flame disturbances varies with the excitation frequency and the location along the flame [34]. Most importantly, they demonstrated that the non-uniformity in flame phase speed can have strong impacts upon the thermoacoustic instability of the combustion system [34]. Most recently, Manoharan and Hemchandra showed that hydrodynamic instabilities could significantly affect the thermoacoustic instability of a step combustor [35]. Through a numerical study, these authors demonstrated that the velocity perturbations caused by hydrodynamic instabilities can perturb the flame heat release and generate thermoacoustic instability [35].

The present survey of literature shows the necessity of including the non-uniformities of flame phase speed into the thermoacoustic low order models. This, in turn, calls for further theoretical development and comparisons between the existing theories and experiments. The present paper aims at responding to this need, through comparing the linear theories of Schuller et al. [23] and Preetham et al. [32] with the experimental results obtained from a forced laminar flame. In particular, the effect of variations in the phase speed of flame disturbances upon the predicted dynamics is investigated. The setup used for the experiment is similar to that employed by Karimi et al. [17]. However, the experimental data presented in this paper are different to that of Karimi et al. [17] and have not been previously reported. In particular, flame dynamics data are provided for a wide range of equivalence ratio and upstream flow velocity. Such a wide extent of operating parameters has not been considered in previous works. It is shown that this leads to the detection of certain trends in agreements between theories and experiment. A suitable platform is, therefore, formed to compare the two theories of Schuller et al. [23] and Preetham et al. [32] with the experimental results. This illustrates their relative strengths and shortcomings and indicates the necessary future theoretical development. It is also noted that, so far, the theory of Preetham et al. [32] has not be subjected to much experimental validations and the present study is the first rigorous experimental validation of this theory.

\section{Experimental method}

\subsection{Experimental set-up}

Figure 1 shows a schematic view of the experimental setup. Two streams of compressed air and gaseous fuel (vaporised propane) were controlled by means of mass flow controllers (MKS models 100B and 1559A). The capacity of air and fuel mass flow controllers were respectively 100 and 6 standard litres per minute with the accuracy of $0.5 \%$ of the full scale. In the experiments reported in this article, the air flow rate was in the range 
of 30-46 and that of fuel was between 2 to 4 standard litres per minute. An error analysis reveals that, considering the accuracy of mass flow controllers, the setup features a maximum error 0.015 in the equivalence ratio. The measured streams were then mixed together in a manifold far upstream of the rig. The mixture was subsequently carried to the rig and entered that through 3 equally spaced injection ports (see Fig. 2). The rig consisted of a plenum, a contraction and a flame holder which allowed different flame configurations. There were several layers of honey-comb and fine mesh inside the plenum which provided a laminar flow at the flame holder. Due to the existence of various mixing agents (e.g. injection, turbulence, pipe bends, honey combs and layers of fine mesh) complete mixing was almost guaranteed. Nonetheless, the uniformity of equivalence ratio at the flame holder was further confirmed by using Combustion Fast Flame Ionisation Detector (Fast FID). The probe was traversed across the flame holder slot, detecting no meaningful variation in the fuel concentration.

A rim stabilised flame holder was used with a $25 \mathrm{~mm}$ rim diameter (Fig. 2). This flame holder produced a conical laminar flame. The flame was in a quartz tube. The total length of the rig was intentionally chosen to be short enough such that the duct acoustics were of a much higher frequency than the expected response of the flame. Underneath of the burner a loud speaker, RCF model L8S800, with the sensitivity of $93 \mathrm{~dB} / \mathrm{W}$ was installed. This speaker was driven with a sine wave voltage signal, generated by a signal generator and amplified by an amplifier (Fig. 1). The frequency of the driving signal was varied from $20 \mathrm{~Hz}$ up to $250 \mathrm{~Hz}$ in increments of 5 or $10 \mathrm{~Hz}$ for the measurement of the flame transfer function.

A long delay (more than an hour) was intentionally imposed between the flame ignition and start of the experiments. Hence, it was ensured that the whole system is under thermal equilibrium. The instantaneous heat release of the flame was inferred by a photomultiplier tube (PMT, Hamamatsu R928). This was installed about $50 \mathrm{~cm}$ away from the flame and masked by a $\mathrm{CH}^{*}$ filter with centre wavelength of $430 \mathrm{~nm}$ and Full Width Half Maximum (FWHM) of $10 \mathrm{~nm}$, in a darkened test cell. There was a small output of the sensor in the absence of the flame in the dark room (dark current). This value was recorded at the start of each measurement and its mean value was then subtracted from the generated PMT signal in the reactive tests. It is commonly accepted that for laminar flames the chemiluminescence is proportional to heat release rate of the flame, i.e. $V_{P M T}^{\prime} / \bar{V}_{P M T}=q^{\prime} / \bar{q}[6,22,23]$. In this relation $\bar{V}_{P M T}$ is the PMT generated voltage. In the absence of acoustic excitation, the PMT voltage was recorded as the flame equivalence ratio was increased from 0.7 to 1 . This trend was then reversed and equivalence ratio was decreased from 1 to 0.7 . A comparison between the recoded values of PMT generated voltage in ascending and descending equivalence ratios, showed negligible hysteresis (almost 0.2\%). Further, after-pulsing of the PMT was evaluated through exposing the sensor to flame for only a short time and recording the generated signal which showed an after-pulsing ratio of $0.35 \%$.

The instantaneous flow velocity was measured $20 \mathrm{~mm}$ upstream of the flame using a hot-wire positioned perpendicular to the mean flow. The hot-wire had a platinum sensing element of $5 \mu \mathrm{m}$ in diameter, and was specially built for this experiment, featuring a curved wire with a radius of roughly $2 \mathrm{~mm}$ (Fig. 3). This allowed installation such that hot-wire supports were almost outside the flow, while the wire itself penetrated into the flow. The set-up provided a reliable measurement of the flow velocity by isolating the effects of the hole on the flow. Further, the flame was not distorted by the wake of the hot-wire. The hot-wire was calibrated in the 
presence of the flame using the known values of air and fuel flow rates from the mass flow controllers. This was to ensure that the possible heat transfer from the flame to the wire has been taken into account. Both hot and cold flow calibrations were conducted. Figure 4 shows the results of these calibrations. In the cold flow test, air flow rate was changed such that the mean velocity varied from about $0.5 \mathrm{~m} / \mathrm{s}$ to $3 \mathrm{~m} / \mathrm{s}$. This showed a reasonably linear behaviour with some deviation at lower velocities (Fig. 4). In the presence of the flame, the calibration was limited to a smaller velocity range. This was due to the restrictions in flame stabilisation at low and high flow velocities. Figure 4 also shows the calibration data for these hot flows for a range of equivalence ratio. The difference between the cold and hot calibrations was due to the heat transfer from the flame and rig. Strong linear behaviour for the hot flows is evident. The calibration curve for each equivalence ratio shown in Fig. 4 was extrapolated to lower and high velocities. The slope of these lines were then used in the postprocessing software to infer the velocity changes from the change in the hot-wire voltage.

The calibration process was repeated at the beginning of each set of experiments. Less than $1 \%$ variation was observed in the slope of the calibration lines due to the changes in the ambient temperature during the experiment. The uncertainty in the velocity measurement is due to that of mass flow controllers and the inaccuracy of hotwire calibration. An error analysis shows that, air and fuel mass flow controllers could generate a maximum error of $1.8 \mathrm{~cm} / \mathrm{s}$ and the variation in hotwire calibration induces around $1 \mathrm{~cm} / \mathrm{s}$ of error. Therefore, a maximum error of around $3 \mathrm{~cm} / \mathrm{s}$ is expected from the velocity measurements. For the results presented in this paper, excitation amplitudes $\varepsilon=\left|u^{\prime} / \bar{u}\right|$ of 0.1 or 0.15 were used.

Pictures of the flame were captured using an ICCD camera (LaVision, FlowMaster3 with image intensifier) with a UV Nikon lens (f\# 4.5) and a gate width of $200 \mu$ s. The flame images were taken at the frequency of 5 $\mathrm{Hz}$ for $40 \mathrm{~s}$ with a spatial resolution in the imaging plane of $0.055 \mathrm{~mm}$. Hot-wire and camera triggering signals were acquired simultaneously. Thus making it possible to mark the moment of capturing each image on the hot-wire signal. Through this, a phase angle was assigned to each flame image. The images were, subsequently, sorted in the ascending order of this assigned phase angle. Hence, complete oscillation cycles, with at least 18 images per cycle, were reconstructed. The voltages generated by the PMT, hot-wire, camera triggering signal and the amplifier were acquired simultaneously at the sampling rate of $16384 \mathrm{~Hz}$ by using a data acquisition card, NI-PCI-6040E, with a resolution of 12 bits. LABVIEW software was used for data logging.

\subsection{Cold flow and steady reactive tests}

A number of cold tests were performed to ensure that uniform mean and oscillating flows were established in the duct upstream of the flame. These non-reactive tests included running the speaker at a constant input voltage and moving the hot-wire (calibrated at one point) across the duct radially. Figures 5 and 6 show respectively the mean value and amplitude of the velocity fluctuations at various duct radii. As can be seen in Fig. 5, the mean velocity is nearly constant across the tested region and shows deviation of less than $3 \%$ of the average velocity. These small changes in the velocity are almost within the limit of the accuracy of the velocity measurements. Figure 6 depicts the amplitude of the velocity fluctuations non-dimensionalised by the local mean velocity. At each position of the hot-wire across the duct, a range of frequencies were tried. As the test 
was performed at a constant speaker voltage, the speaker dynamics are apparent in this figure. In reactive tests, however, the input voltage was varied at each excitation frequency to produce a constant amplitude velocity oscillation. The results particularly those obtained at frequencies above $50 \mathrm{~Hz}$ show that for most of the duct radius, the amplitude of velocity fluctuations is reasonably constant regardless of the probe's location, when it was located more than $1 \mathrm{~mm}$ away from the wall (i. e. $r / \mathrm{R}>0.08$ ). For the measurements made farther than 2 $\mathrm{mm}$ from the internal walls, the responses at various locations are very similar and deviation is less than $4 \%$ of the farthest measurement. An exception to this is a part of the low frequency region (frequencies around 50 $\mathrm{Hz}$ ). This is not surprising as this region is almost outside the audible frequency and therefore the speaker performance is expected to degrade.

Table 1 shows the operating conditions of the experimental data sets presented in this paper. This table also includes the measured values of laminar flame speed and inferred values of the flame cone angle and length under steady state conditions. The standard method of flame cone angle was used for these measurements [36]. Flame cone angle was calculated from the images of steady flame at different equivalence ratios. Kinematic relationships were then used to relate the unstretched laminar flame speed to the flame cone angle and length [36].

In the selection of the experimental conditions detailed in Table 1, the following points were considered. First, the low forcing amplitude cases studied previously had a relative forcing amplitude of $15 \%$ (see Table 2). Karimi et al. [17] investigated the effects of increasing this amplitude. However, lower forcing amplitudes remained unstudied. Current investigation extends the relative forcing amplitudes to $10 \%$ and examines the results. Second, as discussed in later sections and also predicted theoretically [23,32], flame length plays an important role in the linear flame dynamics. Flame length is, in turn, dependent upon equivalence ratio and cold flow velocity. To further explore these effects, a wider range of cold flow velocity and more equivalence ratios are investigated in this paper. Third, recent numerical simulations $[34,39]$ have shown that flame stretch effects can significantly modify the flame dynamics. Yet, these are currently excluded from the low order models $[23,32]$. These effects vary with the equivalence ratio and flow velocity. The current work aims to extend the previous dataset (Table 2) to support future numerical and theoretical studies of flame stretch effects. Finally, the present study closely examines the validity level of the existing low order models [23,32] in a wide range of operating parameters. It is noted that such, comprehensive, comparative analysis has not been previously reported.

\subsection{Post processing of the experimental data}

Calculation of the experimental flame transfer function was based on a linear correlation technique. For a linear single input/output system $H$, with input $U(t)$ and output of $Q(t)$ the frequency response function is (see Fig.7 and [37])

$$
H(f)=\frac{G_{Q U}(f)}{G_{U U}(f)} .
$$


The terms $H, f, U$ and Q in Eq. (3) are respectively the frequency response function, frequency, system input

(normalised velocity, ${ }^{\prime} / \bar{u}$ ) and output (normalised heat release, ${ }^{\prime} / \bar{q}$ ), while $G_{U U}$ and $G_{Q U}$ are the auto-spectral density and cross-spectral density functions [37]. The frequency response function, $H$, is the flame transfer function defined in the left-hand-side of Eqs. (1) and (2). To calculate the flame transfer function, an appropriate length of the hot-wire and PMT signals was first selected. This was generally $7 \mathrm{~s}$ for cases above $30 \mathrm{~Hz}$ and $10 \mathrm{~s}$ for those below this frequency. The fluctuations in the hot-wire voltage were converted to fluctuations in velocity by the hot-wire calibration curve and the mean velocity was measured with the mass flow controllers. The mean heat release was found by averaging the PMT signal. The right-hand-side of Eq. (3) was calculated using the CPSD (Cross Power Spectral Density) command in MATLAB for a given forcing frequency. To check the validity of this method an analytical test case was developed. Two artificial signals were generated with the same sampling frequency $(16384 \mathrm{~Hz})$ and duration $(7 \mathrm{~s})$ as the experimental data. These were $x(\mathrm{t})=\sin$ $(2 \pi f \mathrm{t})$ and $y(\mathrm{t})=a \sin (2 \pi f \mathrm{t}+\pi a)$ where $a$ is a parameter with an initial value of 1 which decreases linearly with a slope of -0.01 per Hz, i.e. $a=1-0.01 f$. Broad band noise was added to these synthetic signals to mimic the signal to noise ratio of the PMT (5-23 dB) and hot-wire (45-47 dB) signals. Further, a secondary signal with an amplitude and phase difference, matching the recorded after-pulse, was added. A linear system with $x$ and $y$ as the input and output has a transfer function with amplitude $a$ and a phase of $\pi a$. The amplitude and phase of the synthetic data was calculated using the software developed for the post-processing of the experimental data. The outcome was almost identical to the analytical transfer function [38].

The amplitude of the transfer function can be also calculated simply by dividing the root-mean-square (RMS) of the output signal by that of the input. The time delay between the PMT and hot-wire signals was calculated using a cross correlation Method [11,37]. The phase of the flame transfer function was then defined as $\phi=2 \pi f \tau$ where $\tau(\mathrm{s})$ is the heat release time delay relative to the upstream velocity forcing [17, 38]. This nonlinear technique was then applied to some of the cases in Table 1. The results were in total agreement with those obtained by the linear method [38]. This confirmed the validity of the assumption of linearity in the postprocessing of the current dataset.

\section{Results and discussion}

\subsection{Comparison with the constant phase speed theory}

Figures 8 to 12 show the measured flame transfer function for all cases presented in Table 1 . They also show the theoretical flame transfer function predicted by the linear kinematic theory of Schuller et al. [23] expressed by Eq. (1). As can be seen in these figures, there is a reasonably good agreement between the amplitude and phase of the calculated and measured flame transfer functions. The agreement is particularly good for the phase response in cases 1, 5 and 7 shown in Figs. 8a, 10a and 11a, respectively. Such phase agreement has not been reported in the previous experimental validations of the linear kinematic theory of Schuller et al. [23] except in Karimi et al [17] for one equivalence ratio $(\varphi=0.9)$ only. Thus, the present results along with those presented in [17] appear to be the best validation of this theory. At lower equivalence ratios, there are some disparities between the measured and calculated flame transfer functions, see Figs. 11b and 12. Other similar experimental 
results [23] also indicate that in general the phase agreement between the experiment and linear theory of Schuller et al. [23] is weaker at lower equivalence ratios.

A close inspection of Figs. 8-11a reveals that at a given equivalence ratio the phase agreement between the theory and experiment improves as the mean cold flow velocity and, therefore, the flame length increases. The amplitude agreement, nonetheless, seems to be quite insensitive to this change. For instance, at equivalence ratio of 0.95 the best phase agreement is seen in Fig. 8a which corresponds to the longest flame amongst cases13. Similarly, between cases 4 and 5 (Figs. 9b and 10a) and, cases 6 and 7 (Figs. 10b and 11a) the longer flame presents a stronger phase agreement between the theory and experiment. Importantly, inclusion of the results of Karimi et al [17] does not change the foregoing argument and for a fixed equivalence ratio the longest flame remains the best match with the experiment. The precise reason for the observed correlation between the phase agreement and flame length is currently unclear. However, a tentative explanation can be put forward by referring to the measurements of Karimi et al. [17]. It was shown in this work that the maximum difference between the measured phase speed and the cold flow convective velocity occurs around the flame base [17]. As the flame disturbance travels along the flame its phase speed approaches that of the cold flow velocity [17]. Hence, the longer the flame is, the average phase speed is closer to the cold flow velocity. It follows that the state of flame disturbances in the long flames better match the theoretical assumptions of constant phase speed, set by the cold flow velocity.

The amplitude of the measured flame transfer function for all investigated equivalence ratios include distinctive peaks and troughs which are not seen in the theoretical transfer function. Observation of this pattern is not limited to the current study and have been also reported by other authors. In a recent experimental study of a laminar conical flame, Cequel et al. [30] showed a series of distinctive peaks and troughs in the amplitude of their measured flame transfer function. A definite explanation for this behaviour of the amplitude does not currently exist. However, existing theoretical evidence [41] suggest that the observed pattern could be due to the dispersion of the convective flame disturbances. The theory of Schuller et al. [23] relates the flame transfer function to the non-dispersive convection of flame disturbances. Theoretical studies [41] on other dynamical systems with dispersive, convective disturbances have shown that the amplitude of the transfer function can feature peaks and troughs. Further, the existing experimental [17] and numerical [34] studies imply the dispersion of flame disturbances. Hence, the observed peaks and troughs in the amplitude of the flame transfer function can be due to dispersion of the flame disturbances. In Figs. 8-12, it also appears that the amplitude agreement with the theory at low forcing frequencies is equivalence ratio dependent. This is such that, in general, the lower equivalence ratio cases feature a better amplitude agreement.

The observed disparities between the theory and experiment could be due to a few reasons. First, the theory essentially ignores the temperature jump across the flame. In reality, however, there is a significant temperature difference between the unburnt and burnt gases. This causes reflection of the incident acoustic wave [40] which in turn intensifies the deviation from the theoretical assumptions. Most importantly, it has been shown that such reflection is more significant at low frequencies $[9,10]$. Therefore, particularly at low frequencies, it sounds plausible to expect the flow field on the flame surface to be more complicated than that assumed by the theory. 
The resultant complication intensifies at higher temperatures and thus it is more pronounced at higher equivalence ratios. It follows that, the existence of this complicated flow can be an explanation for the differences between the measured and predicted flame transfer function at low frequencies. Further, exothermicity of the flame can activate hydrodynamic instabilities of Darrieus-Landau type [39]. It has been shown that these intrinsic instabilities contribute to the dynamics of flame heat release [39] while their strength is dependent upon the temperature ratio of the burnt and unburnt gases [42]. This ratio is equivalence ratio dependent and smaller for leaner flames. Finally, the theory completely ignores the effect of stretch and curvature on the flame speed $[42,43]$. It also assumes zero flame thickness and ignores the variation of flame thickness with changes in equivalence ratio. The latter is expected to contribute to the observed phase disagreement at lower equivalence ratios. Thicker flames at low equivalence ratios can render more involved reactive flow field. This, in turn, can affect the convection of flame disturbances which is an essential parameter affecting the phase of flame transfer function.

Overall, from the dependency of the agreement upon the equivalence ratio, it is speculated that the diffusion effects can substantiate the dipartites between the experiment and theory. The interactions between various mechanisms (complex flow field, hydrodynamic instability and, flame stretch and finite thickness) can cause complexities and invalidate some of the assumption made by the theory. The analysis in the following section shows that releasing the assumption about the constancy of the flame disturbance phase speed can improve the agreement between the theory and experiment.

Figure 13 shows instantaneous images of the flame during the acoustic forcing cycle at three different frequencies. It is clear from this figure that the response of the flame to acoustic excitation includes formation of a flame disturbance at the flame base which then convects along the flame. At high enough frequencies, Fig. 13a, the excited flame features several convecting disturbances and, for the experimental conditions of Fig. 13a and 13b includes flame pinch off $[17,19]$. Decreasing the forcing frequency elongates the wavelength of the flame disturbance and results in a less wrinkled flame, (Figs. 13b and c). As discussed in other works [17, 23 ] these convecting features of the flame dominate the phase response of the flame transfer function. It should be noted that Fig. 13 shows that the flame remains anchored to the flame holder during acoustic excitation. As a result, in this linear limit, the flame disturbances start their growth from zero amplitude at the base of the flame. Flame anchoring is a basic assumption in deriving Eqs. (1) and (2). However, other experimental studies have already shown that this is not the case at higher amplitudes of excitation [17, 31].

\subsection{Comparison with the variable phase speed theory}

Theory of Preetham et al. [32] allows for the flame disturbance phase speed to be freely set. This is done through variation of parameter $\eta$ in Eq. (2). The parameter $\eta$ may change by varying the steady flame length and phase speed of the flame disturbance. Steady flame length is a function of equivalence ratio and mean flow velocity and is a constant for each investigated case (see Table 1). Thus, $\eta$ is changed solely by varying the phase speed. Since in Eq. (2) $u_{0}$ is a constant, $u_{c}$ (the disturbance phase speed) is inversely proportional to $\eta$. It should be noted that the kinematic theory of Preetham et al. [32] assumes that $\eta$ is frequency and location independent. 
It can be, further, readily shown that for $K=1$ this theory reduces to that of Schuller et al. [23]. It is also trivial to demonstrate that $\mathrm{St}_{2}$ in this theory and $\mathrm{St}$ in Eq. (1) are the same, and therefore the non-dimensional frequencies used in Figs. 8-12 and 14-16 are identical.

Figures 14, 15 and 16 depict comparisons between some of the experimental results shown in Figs. 8-12 and the theoretical predictions of Preetham et al. [32] at different values of the phase speed. The comparison is done through varying the parameter $\eta$. The values of $\eta$ and its range of variation were chosen by considering the measured and computed phase speeds reported by Karimi et al [17] and Kashinath et al. [34]. These authors determined the values of $\boldsymbol{u}_{\boldsymbol{c}}$ at the operating conditions similar to those in Tables 1 and 2. Through referring to these studies [17,34] a range of variation for $\boldsymbol{u}_{\boldsymbol{c}}$ was detected. A corresponding variation range was, subsequently, assigned to $\eta$ in Eq. (2). Physically, the phase speed of flame disturbances is determined by the reactive flow field near the flame surface. In an acoustically excited flame, multidimensional and unsteady fluid mechanics as well as the diffusion of heat and mass, highly complicate this flow field. These effects are dependent upon the operating conditions and the forcing frequencies. It is, therefore, not surprising that the phase speed varies with changes in the operating conditions. In high order modelling of flame dynamics, the flame phase speed and its variations can be accurately computed through solving the governing equations [34]. However, since the essential physics of the phase speed alterations are not understood yet, rigorous low order modelling of the variations in phase speed is not currently possible. Hence, these variations can be only included as an input to the low order flame model [32].

Figures 14, 15 and 16 show that for the values of the phase speed greater than the mean velocity of the cold flow $(\eta=0.7$ and 0.8 ) the amplitude of the measured flame transfer function is over predicted for low frequencies $(\mathrm{St}<10)$. However, for these values of the phase speed and for the rest of the investigated frequency range, the amplitude becomes either well predicted or under predicted at the higher frequencies. In this limit of the phase speed $(\eta<1)$, the theoretical predictions of the phase are generally less than the measured ones at the low frequencies $(\mathrm{St}<10)$. Nonetheless, for $\eta<1$ the rest of the frequency range $(\mathrm{St}>10)$ includes a transition in phase agreement. That is for $\varphi=0.95$ and 0.9 in Fig. 14, a good match between the theoretical and experimental phase is seen at $\eta=0.8$. The agreement slightly diminishes at lower equivalence ratios ( $\varphi=0.85$ in Fig. 15a) and the experimental phase starts to shift towards the theoretical prediction for $\eta=0.7$. This trend leads to a relatively good agreement between the experimental and theoretical phases at $\eta=0.7$ for $\varphi=0.78$ and 0.74 (Figs. 15b and 16). Increasing the value of $\eta$ to one and higher leads to major improvements in amplitude prediction at low frequencies $(\mathrm{St}<10)$ for all investigated equivalence ratios, Figs. 14-16. However, the level of agreement drops significantly at higher frequencies.

The same qualitative behaviour applies to the phase of the flame transfer function at the low frequency limit. However, there seems to be a systematic trend in the phase agreement with the theoretical prediction with $\eta \geq$ 1. For the highest equivalence ratio $(\varphi=0.95)$ in Fig $14 a$, the phase agreement with $\eta=1$ remains relatively good 
for the entire frequency range. For the values of $\eta$ greater than one the agreement is good up to $S t \sim 25$. Nonetheless, it is clear in Figs. 14b, 15 and 16 that at lower equivalence ratios, the theoretical and experimental phase agreements are limited to lower Strouhal numbers. This limit is pushed back as the equivalence ratio decreases. As a result, at the lowest equivalence ratios (cases 8 and 9 shown in Fig. 15b and 16) there are poor agreements between the theoretical predictions at $\eta \geq 1$ and experimentally measured phases. The very low frequency part $(\mathrm{St}<5)$, however, remains as an exception and features a general disagreement with the theories of Schuller et al. [23] and Preehtham et al. [32] for all values of the phase speed.

Overall, through varying the phase speed within the designated range the measured amplitude and phase can be predicted for a limited range of frequency. For a given phase speed, simultaneous good phase and amplitude agreements between the experiment and theory can be only achieved for a part of the frequency range. Extension of this agreement to other frequencies requires altering the value of the phase speed. This is to be expected as the previous experiments [17] and computations [34] have shown that the phase speed varies considerably with the forcing frequency. However, the theory of Preetham et al. [32] ignores this frequency dependence. In general, for low to intermediate frequencies the flame dynamics can be fairly predicted by the values of phase speed smaller than that of the cold flow velocity $(\eta \geq 1)$. For higher frequencies, however, using greater values of the phase speed with respect to the cold flow velocity $(\eta<1)$, leads to better agreements between the theory and experiment.

In comparison between the two kinematic theories, it could be argued that, generally, the variable phase speed theory of Preetham et al. [32] gives a better estimation of the amplitude. This is particularly the case at lower frequencies in which the constant phase speed theory features considerable error. Further, the constant phase speed theory [23] sometimes fails to correctly predict the phase of the flame transfer function. This shortcoming is more pronounced at higher frequencies and lower equivalence ratios. In contrast, the variable phase speed theory can provide a good estimation of the phase of the transfer function for all equivalence ratios. Similar to the constant phase speed theory, the variable phase speed theory over predicts the phase at very low frequency range $(\mathrm{St}<5)$.

The analyses in this section clearly show the potential benefits of the variable phase speed models over those assuming constant phase speed. The deviations of the constant phase speed model from the experiment can be significantly reduced by letting the phase speed to vary. However, in its current state, the variable phase speed model requires the numerical value of the phase speed as an input parameters. This adds to the complexity of the problem and requires some knowledge of the flame dynamics. It was shown that, broadly, at low to intermediate frequencies the appropriate phase speed value is slightly smaller than the cold flow velocity. However, to maintain the good agreement between the theory and experiment at higher frequencies, the phase speed should be assigned a value higher than the mean flow velocity. These limitations and complexities indicate that in order to make this theory practically useful, the frequency dependency of the phase speed should be included. This, in turn, requires an understanding of the essential physics responsible for the variation of the phase speed, which calls for further investigations. 


\section{Conclusions}

The transfer function of a conical, ducted, laminar premixed flame was measured experimentally and the results were compared with two existing linear kinematic theories. The experiment was conducted over a range of equivalence ratios and upstream cold flow velocities. The results complemented those reported previously [17] and generally featured a decent agreement with the kinematic theories [23, 32]. Nevertheless, there were some disparities in both phase and amplitude. It was shown that at any equivalence ratio, the phase agreement with the theoretical predictions of Schuller et al. [23] correlates with the flame length. Variable phase speed theory could capture the details of the measured flame dynamics. However, for any given value of the phase speed, this was limited to a certain segment of the frequency range. To maintain a similar level of agreement for the rest of the frequency range, different values of the phase speed should be selected. In general, it was observed that, to have a good agreement between the theory and experiment, the selected values of the phase speed should decrease as the excitation frequency increases. This clearly showed the need for the development of variable phase speed theories that consider the frequency dependency of the phase speed.

\section{References}

[1] Jiménez-Espadafor Aguilar, F., Quintero, R. R., Trujillo, E. C., García, M. T. Analysis of regulation methods of a combined heat and power plant based on gas turbines. Energy, 2014; 72:574-589.

[2] Gobbato P., Masi M., Toffolo A., Lazzaretto A., Tanzini G. Calculation of the flow field and NOx emissions of a gas turbine combustor by a coarse computational fluid dynamics model. Energy, 2012; 45:445-455.

[3] Candel S., Durox D., Schuller T., Darabiha N., Hakim L., Schmitt T. Advances in combustion and propulsion applications, Eur. J. Mechanic. B-Fluid, 2013; 40: 87-106.

[4] Lieuwen T., Yang V. Combustion Instabilities in Gas Turbine Engines. Operational Experience, Fundamental Mechanisms, and Modeling, Progress in Astronautics and Aeronautics, AIAA, Reston, United States, 2005 (chapters $9 \& 12$ ).

[5] Lieuwen, T. C. Unsteady combustor physics. Cambridge University Press, New York, United States, 2012.

[6] Candel S., Durox D., Ducruix S., Birbaud A. L., Noiray N., Schuller T. Flame dynamics and combustion noise, progress and challenge, Int. J. Aeroacoustics, 2009; 8: 1-56.

[7] Zhao, D., Ji, C., Li, S., \& Li, J. Thermodynamic measurement and analysis of dual-temperature thermoacoustic oscillations for energy harvesting application. Energy, 2014; 65: 517-526.

[8] Driscoll J. F. Turbulent premixed flame combustion, Flamelet structure and its effect on turbulent burning velocities, Prog. Energ. Combust. Sci., 2008; 34: 91-134.

[9] Karimi N., Brear M. J., Moase W.H., Acoustic and disturbance energy analysis of a flow with heat communication, J. Fluid Mech., 2008; 597: 67-89.

[10] Karimi N., Brear M. J., Moase W. H. On the interaction of sound with steady heat communicating flows, J. Sound Vib., 2010; 329: 4705-4718.

[11] Hield P.A., Brear M. J. Comparison of Open and choked premixed combustor exits during thermoacoustic limit cycle, AIAA J., 2008; 46: 517-526. 
[12] Hield P.A., Brear M. J., Jin S. H. Thermoacoustic limit cycles in a premixed laboratory combustor with open and choked exits ,Combust. Flame, 2009; 156: 1683-1697.

[13] Kabiraj L., Pankaj W. and Sujith R. I., Bifurcations of self-excited ducted laminar premixed flames., J Eng Gas Turb Power, 2012; 134(3): 031502.

[14] Kabiraj L., and Sujith R. I. Nonlinear self-excited thermoacoustic oscillations: intermittency and flame blowout, J. Fluid Mech. 2012; 713: 376-397.

[15] Kashinath K., Hemchandra S., and Juniper M. P. Nonlinear phenomena in thermoacoustic systems with premixed flames, J. Eng. Gas Turb. Power, 2013;135(6): 061502.

[16] Wu, F., Shu, A., Guo, F., \& Wang, T. Thermoacoustic oscillation basing on parameter exciting. Energy, 2014; 68: 370-376.

[17] Karimi N., Brear M.J., Jin S.H., Monty J.P. Linear and non-linear forced response of a conical, ducted, laminar premixed flame, Combust. Flame, 2009; 156: 2201-2212.

[18] Talei M., Brear M. J., Hawkes E. R. A parametric study of sound generation by laminar premixed flame annihilation, Combust. Flame, 2012; 159: 757-769.

[19] Talei M., Brear M. J., Hawkes E. R., Sound generation by laminar premixed flame annihilation, J. Fluid Mech., 2011; 679: 194-218.

[20] Talei M., Brear M. J., Hawkes E. R. A direct numerical simulation study of frequency and Lewis number effects on sound generation by two-dimensional forced laminar premixed flames, Proc. Combust Inst., 2013; 34: 1093-1100.

[21] Shin D., Lieuwen T., Flame wrinkle destruction processes in harmonically forced, turbulent premixed, J. Fluid Mech., 2013; 721: 484-513.

[22] Ducruix S., Durox D., Candel S. Theoretical and experimental determinations of the transfer function of a laminar premixed flame, Proc. Combust. Inst., 2000; 28: 765-773.

[23] Schuller T., Durox D., Candel S. A unified model for the prediction of laminar flame transfer functions: comparisons between conical and V-flame dynamics, Combust. Flame, 2003;134: 21-34.

[24] Talei, M., Brear, M. J., Hawkes, E. R. A comparative study of sound generation by laminar, combusting and non-combusting jet flows. Theor. Comp. Fluid. Dyn., 2014; 28(4): 385-408.

[25] Fleifil M., Annaswamy A.M., Ghoneim Z.A., Ghoniem A.F. Response of laminar premixed flame to flow oscillations: a kinematic model and thermoacoustic instability results, Combust. Flame, 1996; 106: 487-510.

[26] Birbaud A. L., Durox D., Candel S., Dynamics of confined premixed flames submitted to upstream acoustic modulations, Combust. Flame, 2006; 146: 541-552.

[27] Birbaud A. L., Durox D., Ducruix S., Candel S., Dynamics of free jets submitted to upstream acoustic modulations, Phys. Fluids, 2007; 19: 013602.

[28] Kornilov V. N., Schreel K. R. A. M., de Goey L. P. H. "Experimental assessment of the acoustic response of laminar premixed Bunsen flames." Proc. Combust. Inst., 2007; 31(1): 1239-1246. 
[29] Kornilov V. N., Rook R., ten Thije Boonkkamp J. H. M., de Goey L. P. H. "Experimental and numerical investigation of the acoustic response of multi-slit Bunsen burners." Combust. Flame, 2009; 156(10): 19571970.

[30] Cuquel A., Durox D., Schuller T. "Scaling the flame transfer function of confined premixed conical flames" P. Combust Inst., 2013; 34: 1007-1014.

[31] Cuquel A., Durox D., Schuller T. "Impact of flame base dynamics on the non-linear frequency response of conical flames" CR Mécanique, 2013; 341(1) : 171-180.

[32] Preetham, Santosh H., Lieuwen T., Dynamics of Laminar premixed flames forced by harmonic velocity disturbances, J. Propuls. Power, 2008; 24: 1390-1402.

[33] Blumenthal, R. S., Subramanian, P., Sujith, R. I., Polifke, W. Novel perspectives on the dynamics of premixed flames. Combust. Flame, 2013; 160(7): 1215-1224.

[34] Kashinath K., Hemchandra S., Juniper M. P. "Nonlinear thermoacoustics of ducted premixed flames:

The influence of perturbation convection speed." Combust. Flame, 2013;160(12): 2856-2865.

[35] Manoharan, K., \& Hemchandra, S. Absolute/Convective instability transition in a backward facing step combustor: Fundamental mechanism and influence of density gradient. J. Eng. Gas Turb. Power, 2014, doi:10.1115/1.4028206.

[36] Turns S. R. An introduction to combustion. New York, United States, McGraw-Hill, 1996.

[37] Bendat J., Piersol A. Engineering applications of correlation and spectral analysis. New York, United States, Wiley-Interscience, 1980.

[38] Karimi N., "On the interaction of sound with combusting flows", $\mathrm{PhD}$ thesis, University of Melbourne, Melbourne, Australia, 2009.

[39] Hartmann, D., Schröder, W., Hemchandra, S. Influence of hydrodynamic instability on the heat release transfer function of premixed flames. ASME 2010, GT 2010-22848.

[40] Santosh, H., Sujith, R. I. Acoustic near-field characteristics of a wrinkled premixed flame. Combust. Sci. Tech., 2006; 178(7): 1263-1295.

[41] Sattelmayer, T. Influence of the combustor aerodynamics on combustion instabilities from equivalence ratio fluctuations. J. Eng. Gas Turb. Power, 2003; 125(1): 11-19.

[42] Law, C. K., Sung, C. J. Structure, aerodynamics, and geometry of premixed flamelets. Prog. Energy Combust. Sci., 2000; 26(4): 459-505.

[43] Wang, H. Y., Law, C. K., Lieuwen, T. Linear response of stretch-affected premixed flames to flow oscillations. Combust. Flame, 2009; 156(4), 889-895. 
Tables:

Table1: Operating parameters and steady flame characteristics, studied in the current work.

\begin{tabular}{|l|l|l|l|l|l|l|}
\hline $\begin{array}{l}\text { Case } \\
\text { number }\end{array}$ & $\begin{array}{l}\text { Equivalence } \\
\text { ratio }(\varphi)\end{array}$ & $\begin{array}{l}\text { Upstream cold } \\
\text { flow velocity } \\
(u)(\mathrm{m} / \mathrm{s})\end{array}$ & $\begin{array}{l}\text { Forcing } \\
\text { amplitude } \\
\varepsilon=\left|u^{\prime} / \bar{u}\right|\end{array}$ & $\begin{array}{l}\text { Measured } \\
\text { laminar } \\
\text { flame speed } \\
(\mathrm{m} / \mathrm{s})\end{array}$ & $\begin{array}{l}\text { Flame } \\
\text { half apex } \\
\text { angle }(\alpha) \\
(\text { degrees })\end{array}$ & $\begin{array}{l}\text { Flame } \\
\text { length } \\
(\mathrm{mm})\end{array}$ \\
\hline 1 & 0.95 & 1.54 & 0.15 & 0.423 & 15.9 & 43.9 \\
\hline 2 & 0.95 & 1.2 & 0.1 & 0.423 & 20.6 & 33.2 \\
\hline 3 & 0.95 & 1.26 & 0.15 & 0.423 & 19.6 & 35.1 \\
\hline 4 & 0.9 & 1.12 & 0.15 & 0.414 & 21.7 & 31.4 \\
\hline 5 & 0.9 & 1.4 & 0.1 & 0.414 & 17.2 & 40.4 \\
\hline 6 & 0.85 & 1.32 & 0.15 & 0.36 & 15.8 & 44.2 \\
\hline 7 & 0.85 & 1.54 & 0.15 & 0.36 & 13.5 & 52.0 \\
\hline 8 & 0.78 & 1.18 & 0.15 & 0.266 & 13.0 & 54.1 \\
\hline 9 & 0.74 & 1.0 & 0.1 & 0.227 & 13.1 & 53.7 \\
\hline
\end{tabular}

Table 2: Operating parameters of the linear cases investigated by Karimi et al. [17]

\begin{tabular}{|c|c|c|c|}
\hline $\begin{array}{l}\text { Case } \\
\text { number }\end{array}$ & $\begin{array}{l}\text { Equivalence } \\
\text { ratio }(\varphi)\end{array}$ & $\begin{array}{l}\text { Upstream } \\
\text { cold flow } \\
\text { velocity } \quad(u) \\
(\mathrm{m} / \mathrm{s})\end{array}$ & $\begin{array}{l}\text { Forcing } \\
\text { amplitude } \\
\varepsilon=\left|u^{\prime} / \bar{u}\right|\end{array}$ \\
\hline 1 & 0.95 & 1.32 & 0.15 \\
\hline 2 & 0.9 & 1.32 & 0.15 \\
\hline 3 & 0.85 & 1.4 & 0.15 \\
\hline 4 & 0.75 & 1 & 0.15 \\
\hline
\end{tabular}


Figures:

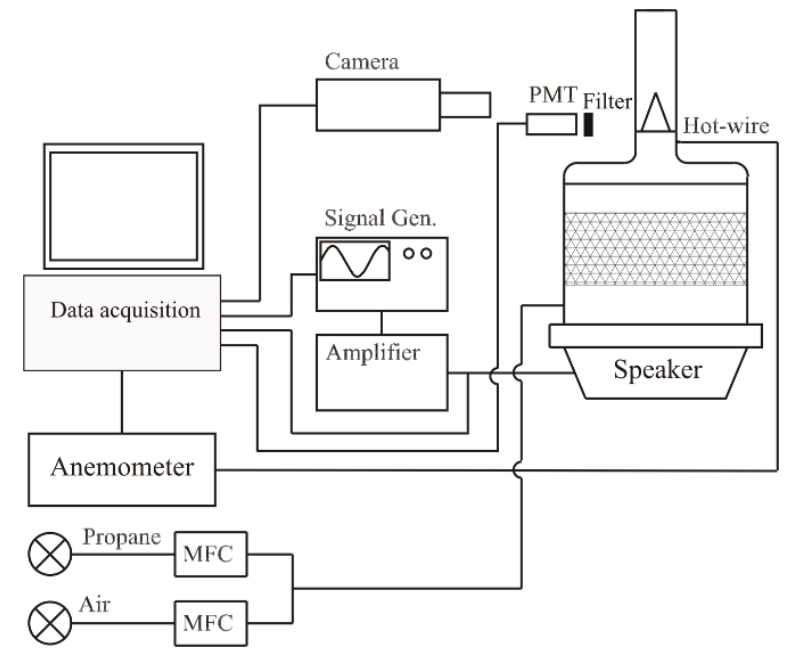

Fig. 1: Schematic of the experimental setup.

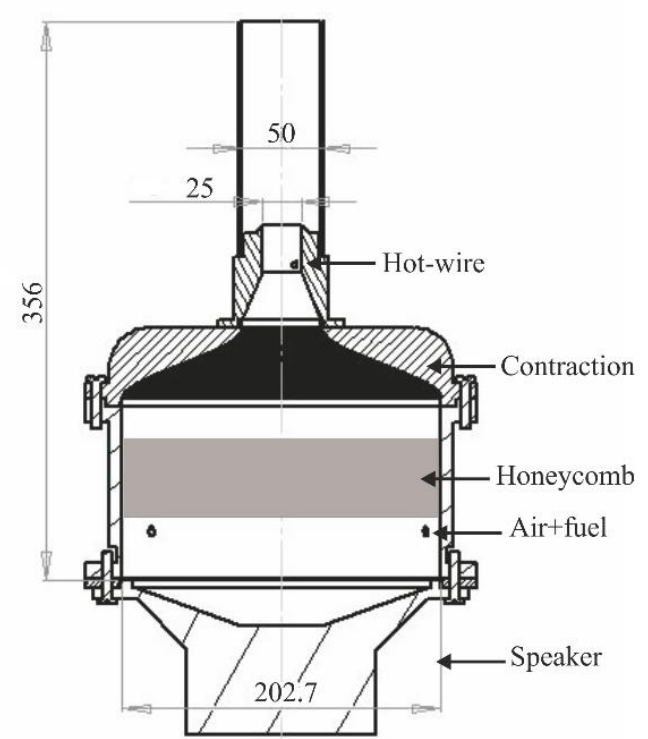

Fig. 2: Section-view of the burner. 


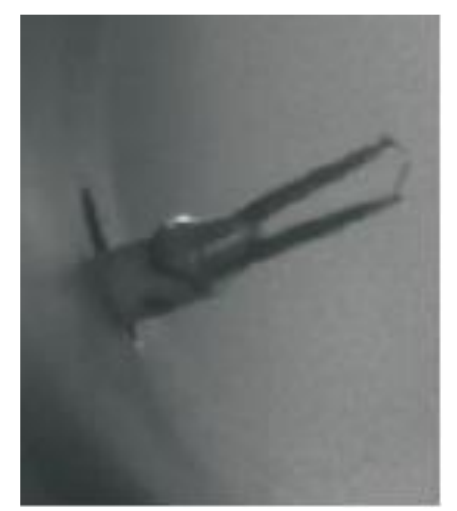

Fig. 3: The hooked hot-wire pushed into the gas passage.

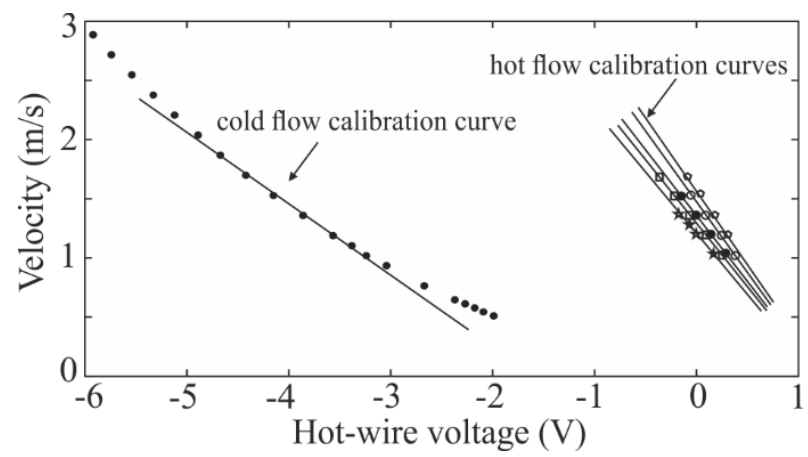

Fig. 4: Calibration curves for cold and hot flows with equivalence ratio of: 0.95 (polygon), 0.9 (circle), 0.85 (solid circle), 0.8 (square), 0.74 (star).

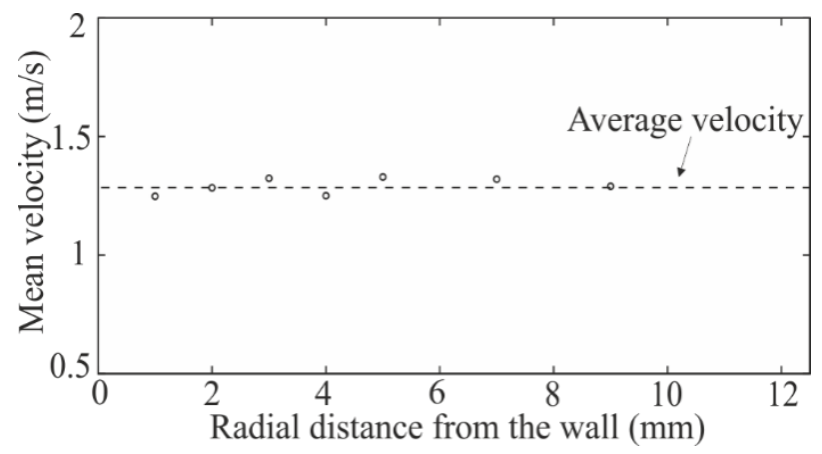


Fig. 5: Mean velocity across the duct, circles: mean velocity from hot-wire, dashed line: average velocity from the mass flow controller, duct radius $\mathrm{R}=12.5$ $\mathrm{mm}, \mathrm{r}=0$ shows the wall and $\mathrm{r}=\mathrm{R}$ is the centre of the duct.

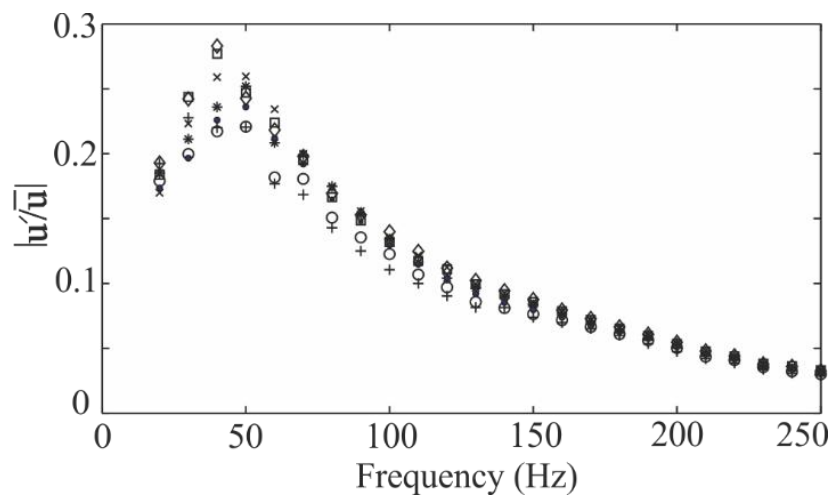

Fig. 6: Amplitude of the velocity fluctuations at varying radial distance against frequency at constant speaker input voltage, $\mathrm{r} / \mathrm{R}=0.08$ (plus), 0.16 (circle), 0.24 (star), 0.32 (dot), 0.4 (cross), 0.56 (square), 0.72 (diamond), $r=0$ shows the wall and $r=R$ is the centre.

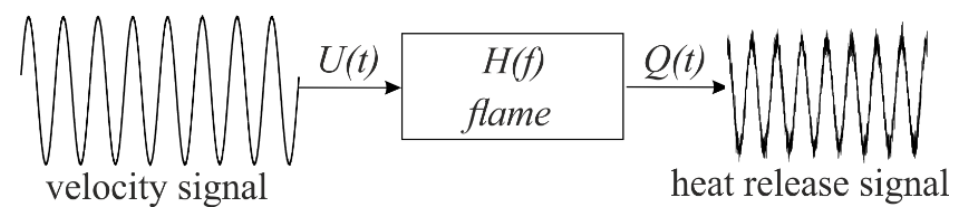

Fig. 7: Black box representation of the post-processing method. 

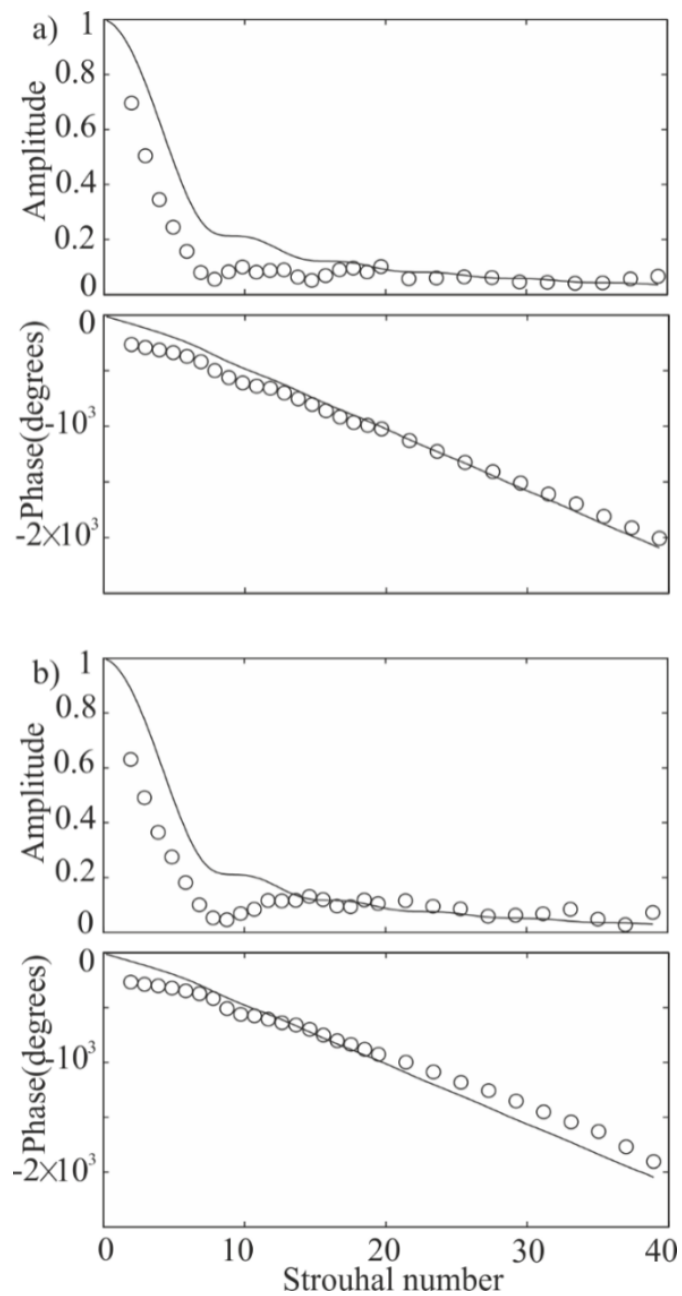

Fig. 8: Flame transfer function, circles: experiments, solid line: Schuller et al. [23] linear theory, a) case1: $\varphi=0.95, \bar{u}=1.53 \mathrm{~m} / \mathrm{s}$ and $\varepsilon=0.15$; and b) case $2: \varphi=$ $0.95, \bar{u}=1.2 \mathrm{~m} / \mathrm{s}$ and $\varepsilon=0.1$. 

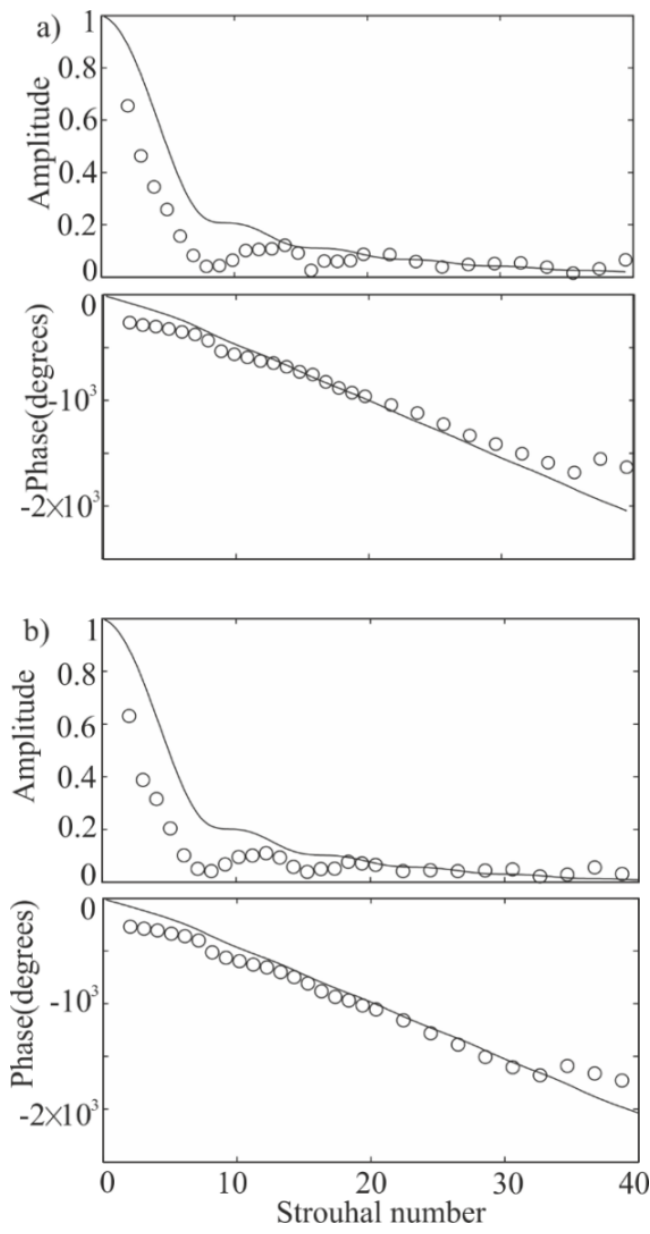

Fig. 9: Flame transfer function, circles: experiments, solid line: Schuller et al. [23] linear theory, a) case $3: \varphi=0.95, \bar{u}=1.26 \mathrm{~m} / \mathrm{s}$ and $\varepsilon=0.15$; and b) case $4: \varphi=0.9, \bar{u}=1.12 \mathrm{~m} / \mathrm{s}$ and $\varepsilon=0.15$. 

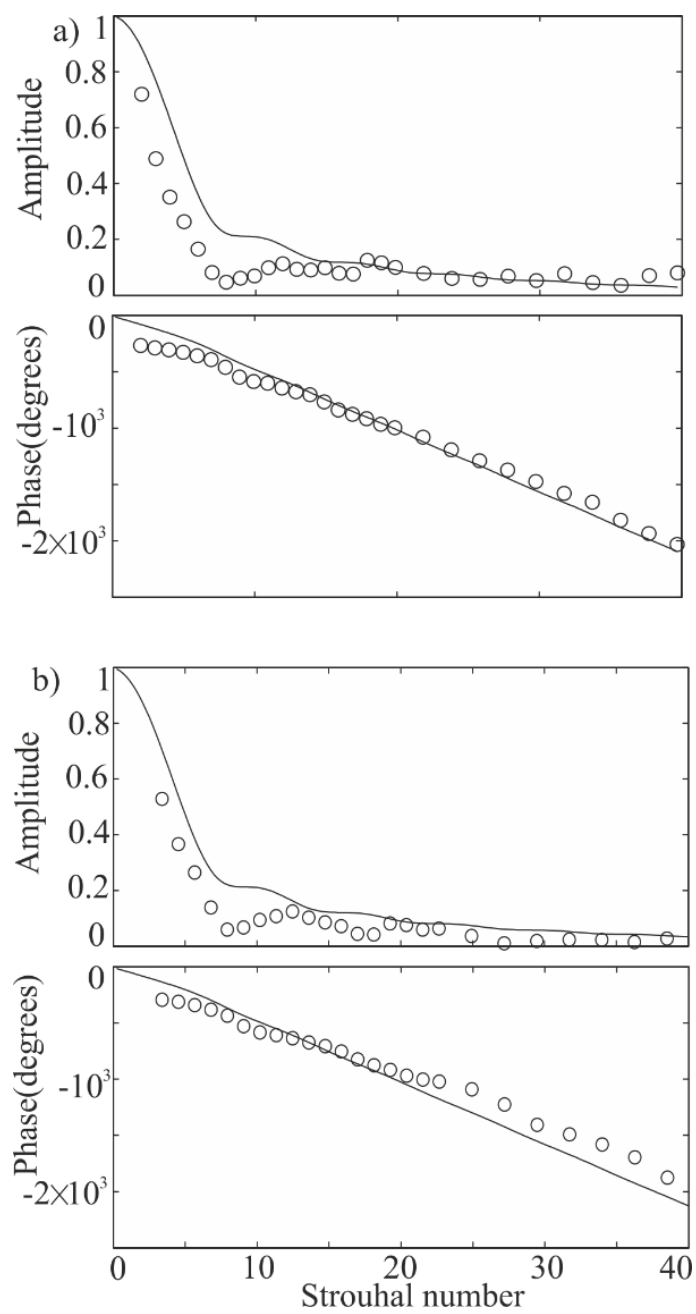

Fig. 10: Flame transfer function, circles: experiments, solid line: Schuller et al. [23] linear theory, a) case 5: $\varphi=0.9, \bar{u}=1.4 \mathrm{~m} / \mathrm{s}$ and $\varepsilon=0.1$; and b) case 6: $\varphi=0.85, \bar{u}=1.32 \mathrm{~m} / \mathrm{s}$ and $\varepsilon=0.15$. 

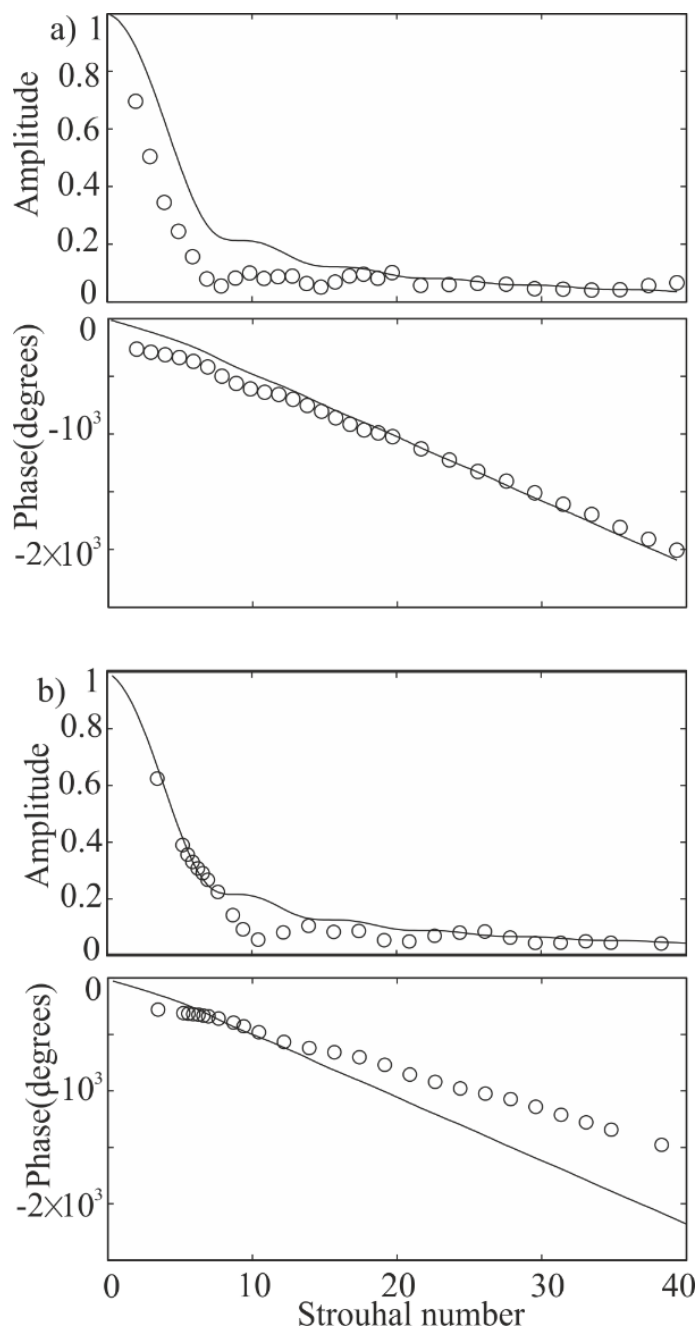

Fig. 11: Flame transfer function, circles: experiments, solid line: Schuller et al. [23] linear theory, a) case 7: $\varphi=0.85, \bar{u}=1.54 \mathrm{~m} / \mathrm{s}$ and $\varepsilon=0.15 ;$ and b) case $8: \varphi=0.78, \bar{u}=1.18 \mathrm{~m} / \mathrm{s}$ and $\varepsilon=0.15$. 

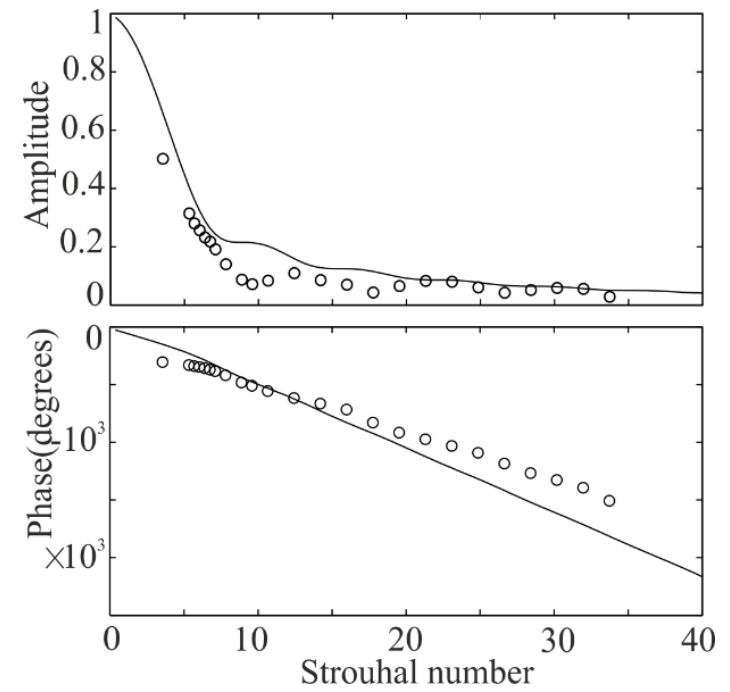

Fig. 12: Flame transfer function, circles: experiments, solid line: Schuller et al. [23] linear theory, case 9: $\varphi=0.74, \bar{u}=1.0 \mathrm{~m} / \mathrm{s}$ and $\varepsilon=0.10$. 
a)

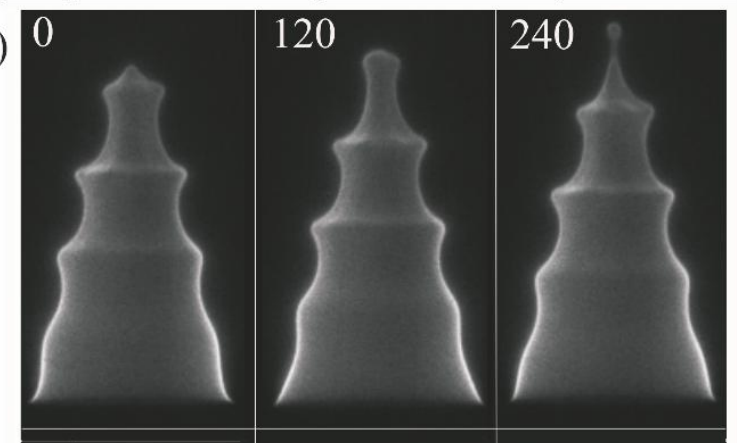

b) 0

120

240

c)
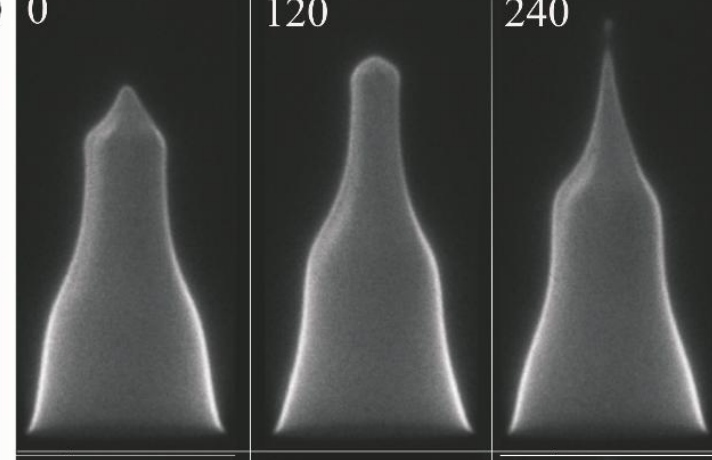

0

120

240

Fig. 13: Instantaneous images of flame during one forcing cycle, $\phi=0.85, \bar{u}=1.32 \mathrm{~m} / \mathrm{s}, \varepsilon=0.15$, a) $S t=29.6$, b) $S t=15$, c) $S t=9$. The numbers on the top left corners, are the phase angles of the oscillations in degrees. 

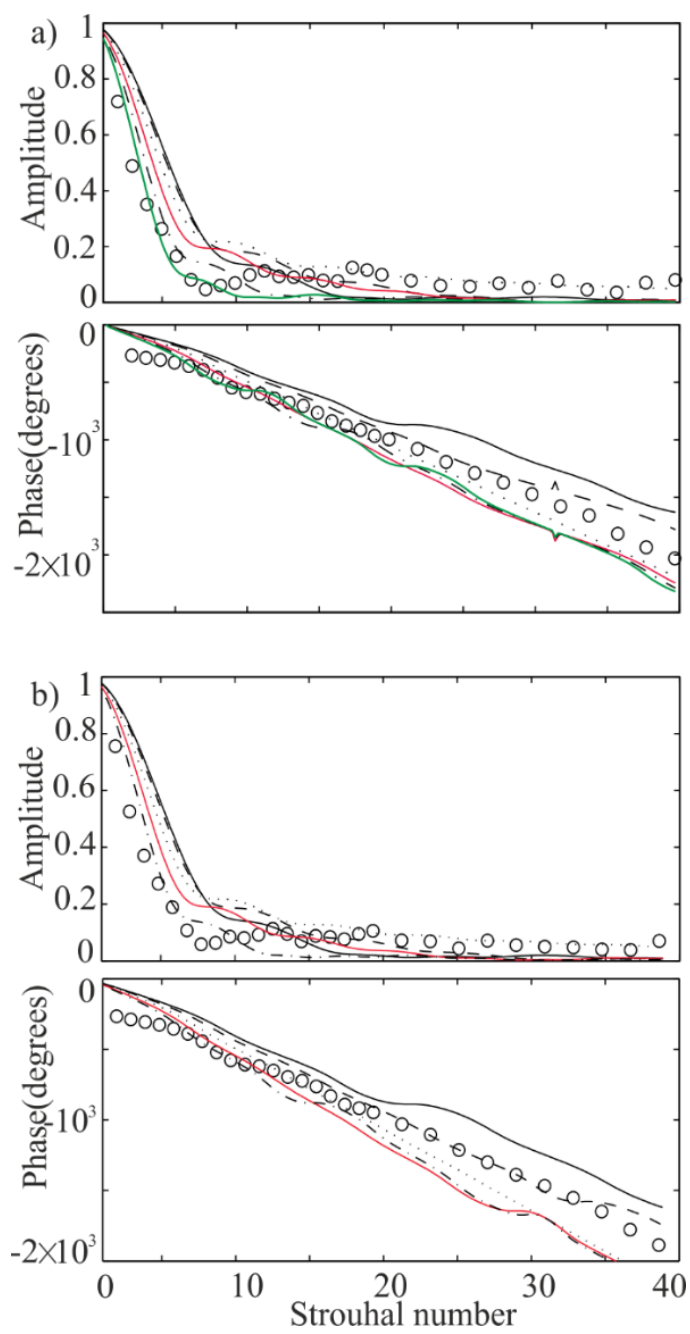

Fig. 14: Flame transfer function, circles: experiments, lines: Preethamet al. [32] linear theory, solid line: $\eta=0.7$, dashed line: $\eta=0.8$, dot line: $\eta=1$, red line: $\eta=1.2$, dot-dash line: $\eta=1.4$, green line: $\eta=1.6$. a) case $1: \varphi=0.95, \bar{u}$ $=1.53 \mathrm{~m} / \mathrm{s}$ and $\varepsilon=0.15, \mathrm{~b})$ case $4: \varphi=0.90, \bar{u}=1.4$ $\mathrm{m} / \mathrm{s}$ and $\varepsilon=0.1$. 

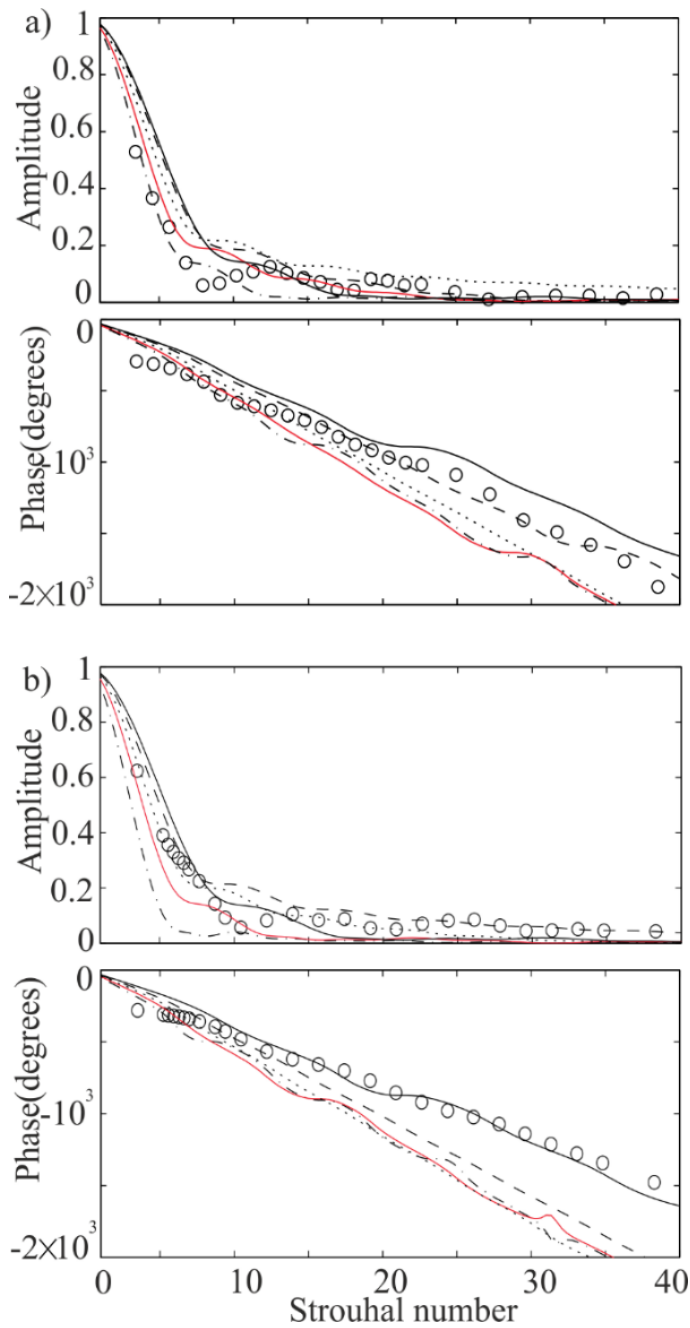

Fig. 15: Flame transfer function, circles: experiments, lines: Preethamet al. [32] linear theory, solid line: $\eta=0.7$, dashed line: $\eta=0.8$, dot line: $\eta=1$, red line: $\eta=1.2$, dot-dash line: $\eta=1.4$. a) case $6: \varphi=0.85, \bar{u}$ $=1.32 \mathrm{~m} / \mathrm{s}$ and $\varepsilon=0.15, \mathrm{~b})$ case $8: \varphi=0.78, \bar{u}=1.18 \mathrm{~m} / \mathrm{s}$ and $\varepsilon=0.1$. 

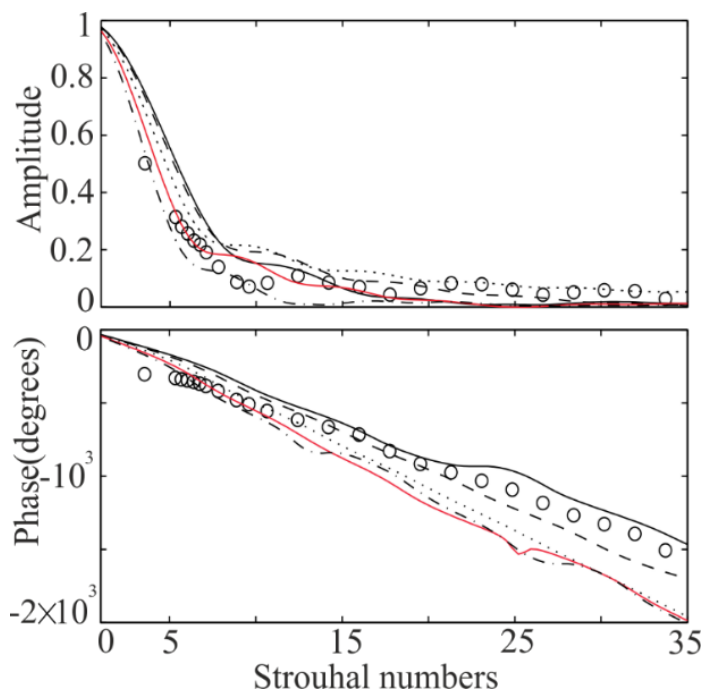

Fig. 16: Flame transfer function, circles: experiments, lines: Preethamet al. [32] linear theory, solid line: $\eta=0.7$, dashed line: $\eta=0.8$, dot line: $\eta=1$, red line: $\eta=1.2$, dot-dash line: $\eta=1.4$. Caser 9: $\varphi=0.74, \bar{u}=1.0$ $\mathrm{m} / \mathrm{s}$ and $\varepsilon=0.1$. 